\title{
Adaptive Feedback Cancellation With Band-Limited LPC Vocoder in Digital Hearing
} Aids

Ma, Guilin; Gran, Fredrik; Jacobsen, Finn; Agerkvist, Finn T.

Published in:

I E E E Transactions on Audio, Speech and Language Processing

Link to article, DOI:

10.1109/TASL.2010.2057245

Publication date:

2011

Document Version

Publisher's PDF, also known as Version of record

Link back to DTU Orbit

Citation $(A P A)$ :

Ma, G., Gran, F., Jacobsen, F., \& Agerkvist, F. T. (2011). Adaptive Feedback Cancellation With Band-Limited LPC Vocoder in Digital Hearing Aids. I E E E Transactions on Audio, Speech and Language Processing, 19(4), 677-687. https://doi.org/10.1109/TASL.2010.2057245

\section{General rights}

Copyright and moral rights for the publications made accessible in the public portal are retained by the authors and/or other copyright owners and it is a condition of accessing publications that users recognise and abide by the legal requirements associated with these rights.

- Users may download and print one copy of any publication from the public portal for the purpose of private study or research.

- You may not further distribute the material or use it for any profit-making activity or commercial gain

- You may freely distribute the URL identifying the publication in the public portal 


\title{
Adaptive Feedback Cancellation With Band-Limited LPC Vocoder in Digital Hearing Aids
}

\author{
Guilin Ma, Student Member, IEEE, Fredrik Gran, Finn Jacobsen, and Finn Thomas Agerkvist
}

\begin{abstract}
Feedback oscillation is one of the major issues with hearing aids. An effective way of feedback suppression is adaptive feedback cancellation, which uses an adaptive filter to estimate the feedback path. However, when the external input signal is correlated with the receiver input signal, the estimate of the feedback path is biased. This so-called "bias problem" results in a large modeling error and a cancellation of the desired signal. This paper proposes a band-limited linear predictive coding based approach to reduce the bias. The idea is to replace the hearing-aid output with a synthesized signal, which sounds perceptually the same as or similar to the original signal but is statistically uncorrelated with the external input signal at high frequencies where feedback oscillation usually occurs. Simulation results show that the proposed algorithm can effectively reduce the bias and the misalignment between the real and the estimated feedback path. When combined with filtered-X adaptation in the feedback canceller, this approach reduces the misalignment even further.
\end{abstract}

Index Terms-Adaptive feedback cancellation (AFC), hearing aids, linear predictive coding (LPC).

\section{INTRODUCTION}

$\mathbf{F}$ EEDBACK in a hearing aid refers to a process in which a part of the receiver output is picked up by the microphone. The acoustic feedback path is the most significant contributor to the feedback signal although electrical and mechanical paths also exist [1]. A typical acoustic feedback path of the hearing aid represents a wave propagation path from the receiver to the microphone, which includes the effects of the hearing-aid receiver, the microphone, the acoustics of the vent or leak, etc. The hearing-aid processing amplifies the input signal to compensate for the hearing loss of the users. When this amplification is larger than the attenuation of the feedback path, instability occurs and usually results in feedback whistling, which limits the maximum gain that can be achieved [2] and compromises the comfort of wearing hearing aids.

A widely adopted approach to acoustic feedback suppression is adaptive feedback cancellation (AFC), in which the acoustic

Manuscript received December 05, 2009; revised March 05, 2010 and May 28, 2010; accepted June 18, 2010. Date of publication July 12, 2010; date of current version February 14, 2011. The associate editor coordinating the review of this manuscript and approving it for publication was Dr. Jingdong Chen.

G. Ma is with Acoustic Technology, Department of Electrical Engineering, Technical University of Denmark, 2800 Kgs. Lyngby, Denmark, and also with GN Research, GN ReSound A/S, 2750 Ballerup, Denmark (e-mail:mguilin@gnresound.dk).

F. Gran is with GN Research, GN ReSound A/S, 2750 Ballerup, Denmark (e-mail: fgran@gnresound.dk).

F. Jacobsen and F. T. Agerkvist are with Acoustic Technology, Department of Electrical Engineering, Technical University of Denmark, $2800 \mathrm{Kgs}$. Lyngby, Denmark (e-mail: fja@elektro.dtu.dk; fa@elektro.dtu.dk).

Digital Object Identifier 10.1109/TASL.2010.2057245 feedback signal is estimated by an adaptive filter and then subtracted from the input signal to remove feedback [3]. A perfect match between the modeled and the real feedback path will cancel the feedback signal completely, and prevent instability for any amount of amplification. However, in practice, there is always a modeling error for many reasons, such as too slow adaptation speed, insufficient filter length, etc. A significant portion of the modeling error is the result of a so-called "bias problem," which refers to a biased estimate of the feedback path when the desired input signal and the receiver input signal are correlated [4]. During the past two decades, various approaches have been proposed to decorrelate the input and output of a hearing aid to reduce the bias in the estimate of the feedback path.

One well-known decorrelation approach introduces a delay in the hearing-aid processing (or the feedback cancellation path) to decorrelate the input of the receiver and the incoming signal. It has been shown in [4] that for a colored noise input, the insertion of delay in the hearing-aid processing significantly improves the accuracy of feedback modeling, while the insertion of a delay in the feedback cancellation path provides smaller benefit. However, the delay introduced in the hearing aids should be kept small to avoid disturbing artifacts such as comb filtering [5]. Moreover, for tonal signals, a delay will not help much to reduce the correlation.

Another kind of decorrelation approach uses nonlinearities in the hearing-aid processing. Methods based on this approach include frequency shifting [6], time-varying all-pass filter [7], etc. Since all the nonlinear methods degrade sound quality to some extent, a tradeoff between the performance of feedback cancellation and sound quality is usually involved.

Alternatively, a probe signal, usually a noise signal, can be added to the receiver input [8]. To maintain sound quality, the probe signal should be inaudible and its level therefore has to be much lower than that of the original receiver input signal. The bias reduction achieved with such a weak probe signal is very small.

A recently proposed decorrelation method exploits closed-loop identification techniques [9]-[11]. In [11], it has been proven that by minimizing the prediction error of the microphone signal, the estimate of the feedback path is not biased (identifiable) when the desired input signal is an autoregressive (AR) random process and when certain conditions are met. A prediction error method-based adaptive feedback cancellation (PEM-AFC) is proposed in [11] to identify the models for the desired signal and the feedback path simultaneously. However, in practice, many desired input signals, such as voiced speech and music, are not AR processes. Moreover, the 


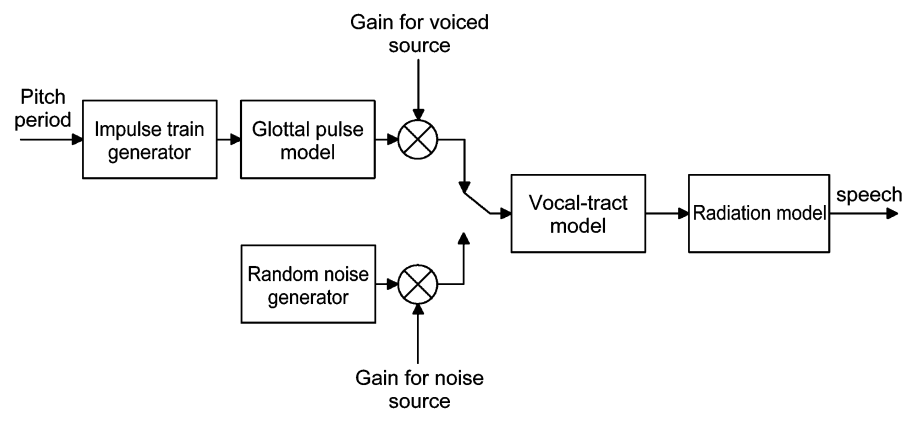

Fig. 1. General discrete-time model for speech production [14].

conditions for identification may often be violated, for example, when insufficient filter length is used for modeling the desired input signal. In these cases, bias remains in the estimate of the feedback path.

This paper proposes a new linear predictive coding based approach for reducing the bias. The idea is to generate a synthetic signal for the receiver input, which sounds perceptually similar to or possibly even the same as the original signal but is statistically uncorrelated with the desired input signal. It is shown that this approach reduces the bias significantly and improves the performance of the feedback cancellation system.

The paper is organized as follows. Section II describes the basic theory of linear predictive coding. In Section III, the band-limited linear predictive coding-based adaptive feedback cancellation (BLPC-AFC) is proposed. An adaptive feedback cancellation system combining the BLPC-AFC and filtered-X adaptation is described in Section IV. In Section V, simulation results are presented and sound quality of the synthetic signals is discussed. Concluding remarks are provided in Section VI.

\section{Linear Predictive Coding}

Parametric representation of a spectrum by means of linear prediction (LP) is a powerful technique in speech and audio signal processing. Linear predictive coding (LPC) was developed for the purpose of speech compression in the 1960s [12]. After that, research on LPC vocoder resulted in the $2.4 \mathrm{~kb} / \mathrm{s} \mathrm{se}$ cure-voice standard LPC10 [13]. However, the sound quality produced by LPC vocoder at low bit rates was not good enough for commercial telephony [12]. To provide high-quality speech at low bit rates, in the 1970s and 1980s, residual excited LPC (RELP), multi-pulse LPC and code-excited LPC (CELP) were proposed to code the residual signal in better ways. The following subsections will briefly describe LPC for speech applications and its basis in the speech production model.

\section{A. Discrete-Time Speech Production Model}

The LPC-based vocoder, such as RELP, multi-pulse LPC, CELP, etc., exploits the special properties of a classical discrete-time model of the speech production process, which is illustrated in Fig. 1. During unvoiced speech activity, the excitation source is flat-spectrum noise, modeled by a random noise generator; during periods of voiced speech activity, the excitation uses an estimate of the local pitch period to set an impulse train generator that drives a glottal pulse shaping filter. The excitation is later filtered by the vocal-tract filter and the lip radiation filter to produce the speech. This model, although not adequate

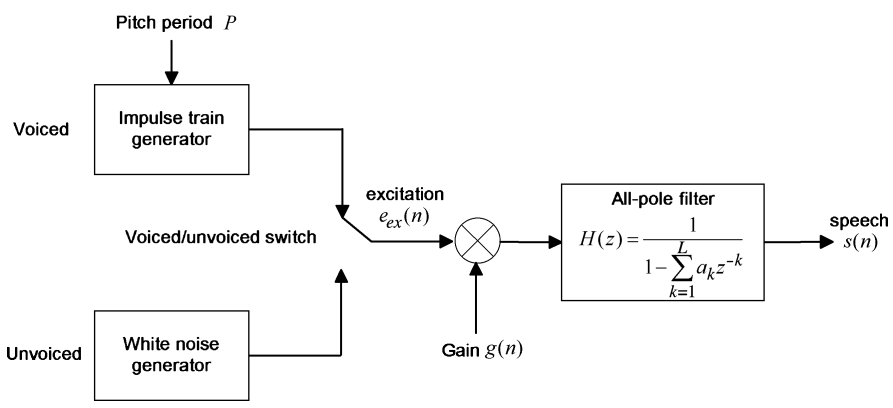

Fig. 2. All-pole model for speech production [14]. The pitch period $P$, the type of excitation, the gain $g(n)$, and the all-pole filter $H(z)$ of order $L$ are parameters to be estimated by linear prediction analysis. The excitation sequence is denoted by $e_{e x}(n)$, and $s(n)$ is the output speech from the production model.

for certain classes of phonemes such as voiced fricatives, has been successfully used in many speech analysis, coding, and recognition tasks.

In general, modeling the transfer functions of vocal tract and lip radiation requires both zeros and poles. However, they can be well approximated by a complete all-pole model as illustrated in Fig. 2, which yields identical magnitude spectra to the true transfer function of the speech production process but might alter the phase characteristics. Applications have justified that correct spectral magnitude is frequently sufficient for coding, recognition, and synthesis [14].

In the all-pole speech model, the output speech $s(n)$ is generated with the excitation sequence $e_{e x}(n)$ in the following way:

$$
\begin{aligned}
s(n) & =\mathbf{a}^{T} \mathbf{s}(n-1)+g(n) e_{e x}(n) \\
\mathbf{a} & =\left[a_{1}, a_{2}, \cdots, a_{L}\right]^{T} \\
\mathbf{s}(n) & =[s(n), s(n-1), \cdots, s(n-L+1)]^{T}
\end{aligned}
$$

where $\mathbf{a}$ is the coefficient vector of the all-pole filter $H(z)$ of order $L$, and the superscript $T$ denotes the transpose of a vector/ matrix.

Equations (1)-(3) suggest that except for the excitation term, $s(n)$ can be predicted using a linear combination of its past $L$ values with the weights $a_{i}$ 's. The $a_{i}$, which characterize the all-pole filter, are usually estimated by an efficient computation technique called linear prediction analysis, which can be done in many ways, for example, by using the autocorrelation methods. The linear prediction analysis will be described in Section II-C.

\section{B. $L P C$ Vocoder}

A typical diagram of LPC-based vocoder is given in Fig. 3. Speech at the coding end $s(n)$ is first analyzed by LP analysis to estimate the set of coefficients of the all-pole filter, the pitch period, the gain parameter and the voiced/unvoiced parameter. These parameters are then encoded. At the decoding end, the speech signal is synthesized in the way illustrated in Fig. 2 using the decoded parameters.

During the estimation of the parameters, the residual signal $s^{\mathrm{p}}(n)$, also referred to as predicted error signal, can be obtained as

$$
\begin{aligned}
s^{\mathrm{p}}(n) & =s(n)-\hat{\mathbf{a}}^{T} \mathbf{s}(n-1) \\
\hat{\mathbf{a}} & =\left[\hat{a}_{1}, \hat{a}_{2}, \cdots, \hat{a}_{L}\right]^{T} \\
\hat{H}(z) & =\frac{1}{1-\sum_{k=1}^{L} \hat{a}_{k} z^{-k}}
\end{aligned}
$$




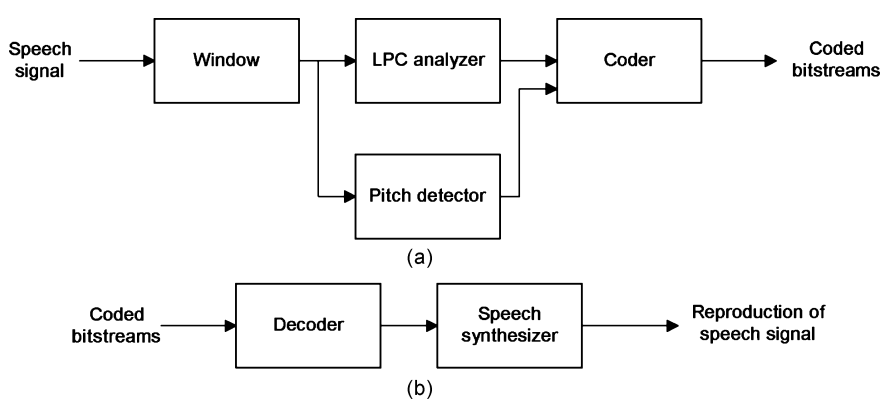

Fig. 3. Block diagram of a typical LPC vocoder: (a) encoder; (b) decoder [15]. The window is typically $10-30 \mathrm{~ms}$ long. The encoded parameters are the set of coefficients computed by LPC analyzer, the pitch period, the gain parameter, and the voiced/unvoiced parameter.

where $\hat{H}(z)$ is the estimated all-pole filter of order $L$, the superscript $\mathrm{p}$ is used to denote the prediction error of the corresponding signal, and the denominator of $\hat{H}(z)$, which represents a finite-impulse-impulse (FIR) filter, is also called the prediction error filter (PEF).

\section{Linear Prediction Analysis}

Linear prediction analysis is a way of estimating the AR model for a given signal. It is usually used in the LPC analyzer (see Fig. 3) to estimate the parameters, such as â. The LP analysis finds the set of coefficients of the all-pole filter by minimizing the mean-squared prediction error: ${ }^{1}$

$$
\hat{\mathbf{a}}_{\mathrm{o}}=\arg \min _{\hat{\mathbf{a}}} E\left\{\left|s^{\mathrm{p}}(n)\right|^{2}\right\}
$$

where $E\{\cdot\}$ is the expectation operator, $s^{\mathrm{P}}(n)$ is the prediction error/residual signal defined in (4), and $\hat{\mathbf{a}}_{\mathrm{o}}$ denote the optimal LP coefficients that minimize the mean-squared error.

Since the speech characteristics vary with time, the all-pole filter coefficients should be estimated by a short-term analysis, which minimizes the mean square of the prediction error over a segment of speech signal. The approaches for short-term LP analysis generally fall into two categories: the autocorrelation method and the covariance method. The autocorrelation method assumes that the samples outside the time segment are all zero. This assumption may result in a large prediction error at the beginning and end of the segment. To taper the segment and deemphasize that prediction error, a window (e.g., a Hamming window) is usually used. The covariance method, on the contrary, makes no assumptions about the values outside the segment and uses the true values.

For the autocorrelation method, the stability of the estimated all-pole filter can be guaranteed, whereas for the covariance method, it cannot be ensured. Therefore, the autocorrelation method is used in this paper. A well-known and efficient way to compute the LP coefficients in the autocorrelation method is through using the Levinson-Durbin recursion algorithm [17], [18].

\footnotetext{
${ }^{1}$ It should be noted that the minimization of the mean-squared prediction error $s^{\mathrm{p}}(n)$ yields an all-pole system $\hat{H}(z)$ modeling the minimum-phase part of the true transfer function in Fig. 1 perfectly only during unvoiced signal segment. For voiced speech, although the model is not exact, the coefficients obtained still comprise a very useful and accurate representation of the speech signal [16].
}

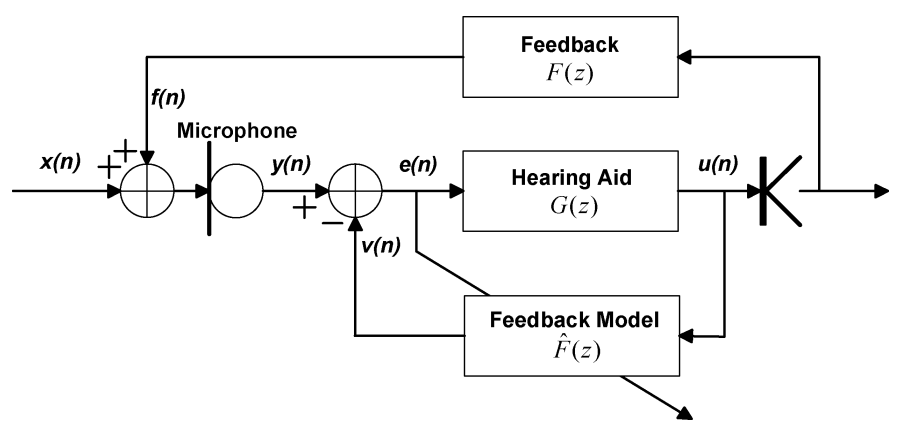

Fig. 4. General diagram of the adaptive feedback cancellation system. The input to the hearing-aid processing is $y(n)$, which is the sum of the desired input signal $x(n)$ and the feedback signal $f(n)$. The hearing-aid process is denoted as $G(z)$, and the processed hearing-aid signal is $u(n)$. The transfer function of the feedback path is $F(z)$, and $v(n)$ is the estimate of $f(n)$ generated by the modeled feedback path $\hat{F}(z)$.

Another special type of methods for linear prediction is the lattice method. A typical lattice method is the Burg Lattice algorithm [19], which also yields stable all-pole filters.

\section{BAND-LIMITED LPC VOCODER FOR AFC}

In this section, the bias problem associated with the AFC is first explained through a steady-state analysis in Section III-A. Next, a new method based on a simplified LPC vocoder is proposed in Section III-B to reduce the bias. The developed LPC vocoder is band-limited to focus on the bias reduction in the critical frequency region of the feedback path and to minimize the impact on sound quality. In the end, the steady-state analysis of the proposed BLPC-AFC is given in Section III-C.

\section{A. Bias Problem With AFC}

A typical block diagram of the AFC is illustrated in Fig. 4. The feedback path model $\hat{F}(z)$ usually consists of an adaptive FIR filter with the vector of coefficients $\hat{\mathbf{f}}$, i.e.,

$$
\begin{aligned}
\hat{F}(z) & =\sum_{k=0}^{L_{\hat{F}}-1} \hat{f}_{k} z^{-k} \\
\hat{\mathbf{f}} & =\left[\hat{f}_{0}, \hat{f}_{1}, \cdots, \hat{f}_{L_{\hat{F}}-1}\right]^{T}
\end{aligned}
$$

where $L_{\hat{F}}$ is the length of the adaptive FIR filter $\hat{F}(z)$.

As pointed out in [4], the adaptation of this FIR filter to minimize the mean square of the error signal $e(n)$ usually leads to a biased estimate when the desired input signal $x(n)$ is correlated with the receiver input signal $u(n)$. This can been shown from the steady-state analysis of the system, during which it is assumed that the feedback path is not varying and the input signal $x(n)$ is a wide-sense stationary process. Suppose that the feedback path $F(z)$ is also an FIR filter with coefficients vector $\mathbf{f}$ and is of the same order as the feedback path model $\hat{F}(z)$. The Wiener solution to the minimization of the mean-square error of the error signal $e(n)$ is

$$
\begin{aligned}
\hat{\mathbf{f}} & =\mathbf{R}_{\mathbf{u u}}^{-1} \mathbf{r}_{\mathbf{u} y} \\
& =\mathbf{f}+\mathbf{R}_{\mathbf{u u}}^{-1} \mathbf{r}_{\mathbf{u} x} \\
\mathbf{R}_{\mathbf{u u}} & =E\left\{\mathbf{u}(n) \mathbf{u}^{T}(n)\right\}
\end{aligned}
$$




$$
\begin{aligned}
\mathbf{r}_{\mathbf{u} y} & =E\{\mathbf{u}(n) y(n)\} \\
\mathbf{u}(n) & =\left[u(n), u(n-1), \cdots, u\left(n-L_{\hat{F}}+1\right)\right]^{T}
\end{aligned}
$$

where $\mathbf{r}_{\mathbf{u} x}$ and $\mathbf{r}_{\mathbf{u} f}$ are defined similarly as in (13). The term $\mathbf{R}_{\mathbf{u u}}^{-1} \mathbf{r}_{\mathbf{u} x}$ in (11) represents the bias of the estimate, which is related to the correlation between the desired input signal $x(n)$ and the processed hearing-aid signal $u(n)$. The magnitude of the bias depends strongly on the decaying speed of the autocorrelation function of $x(n)$, the forward-path delay, and nonlinearity in the hearing-aid process $G(z)$. The bias problem is particularly serious when the desired input signal $x(n)$ is tonal because the correlation function does not drop off.

\section{B. Band-Limited LPC Vocoder for AFC}

To reduce the bias, several approaches have been proposed as mentioned in Section I. Here, a new method to decorrelate $x(n)$ and $u(n)$ using a band-limited LPC is proposed.

The main idea is to create a synthetic replica of the processed hearing-aid signal $u(n)$, which is statistically uncorrelated with $x(n)$ but still sounds perceptually close or identical to $u(n)$. To achieve this, a simplified LPC vocoder is adopted, which consists of three steps: First, LP analysis is performed on $u(n)$ to estimate the all-pole model for $u(n)$; then the residual signal is replaced with a white noise sequence of the same variance as the residual signal of $u(n)$; in the end, the noise sequence drives the obtained all-pole system to synthesize a new signal $u_{\text {syn }}(n)$ for the receiver to output, which maintains the magnitude spectrum of $u(n)$ but is uncorrelated with $x(n)$.

Compared with a standard LPC vocoder, such as LPC10, this simplified vocoder has a great advantage in terms of computation load since it does not need any voiced activity detection and pitch estimation. It also removes the long-term bias in the adaptation completely since $u_{\mathrm{syn}}(n)$ is uncorrelated with $x(n)$ [cf. (11)]. However, as mentioned in Section II, voiced speech is synthesized with an impulse train. ${ }^{2}$ With only white noise driven, the synthesis of voiced speech will degrade sound quality significantly.

To circumvent this issue, a band-limited LPC vocoder (BLPC) is proposed based on the characteristics of the feedback path and the performance of the AFC in practice. Previous research has shown that the magnitude of the frequency response of the feedback path is usually much higher in the region above $2 \mathrm{kHz}$ than that below $2 \mathrm{kHz}$ [20] (cf. Fig. 7 in Section V). For most hearing-aid users, the prescribed forward-path gain is also higher at high frequencies than at low frequencies. Therefore, in practice, the AFC fails to prevent whistling at high frequencies in most cases. Moreover, since the feedback is usually very weak at low frequencies, special methods can be used in the AFC to prevent whistling resulted by the bias at low frequencies. For example, high-pass filters can be used in front of the adaptation of the feedback model to reduce the effect of the bias at low frequencies [21]. Thus, the bias problem is prominent mainly at high frequencies, and the reduction of bias, as a means to improve the performance of the $\mathrm{AFC}$, is mainly needed in the region above $2 \mathrm{kHz}$.

\footnotetext{
${ }^{2}$ Strictly speaking, a phase altered version of an impulse train.
}

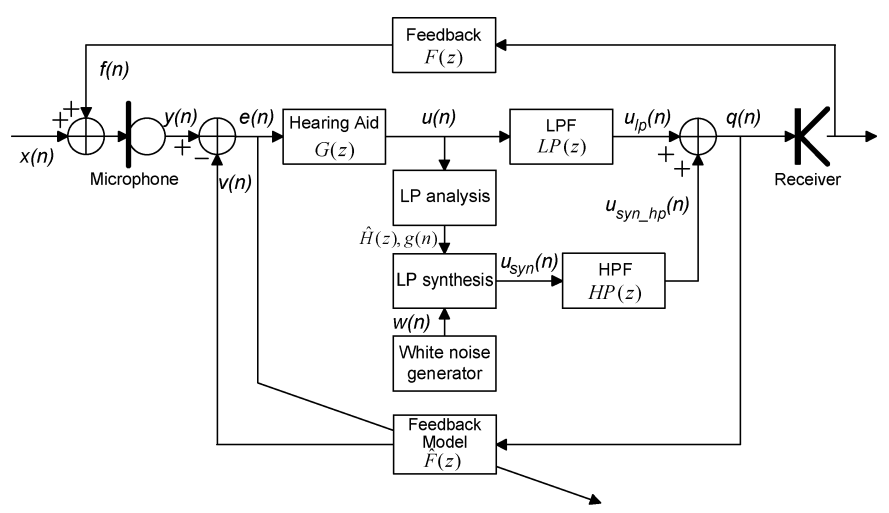

Fig. 5. Diagram of adaptive feedback cancellation with band-limited LPC vocoder. LPF is the low-pass filter with the transfer function LP(z), and HPF is the high-pass filter with the transfer function $\mathrm{HP}(\mathrm{z})$.

To decorrelate $x(n)$ and $u(n)$ at high frequencies, the synthesized signal $u_{\text {syn }}(n)$ is only needed in the high-frequency region while the low-frequency part of the original signal $u(n)$ can be maintained without any modification. This consideration results in a band-limited LPC vocoder-based AFC (BLPC-AFC) as illustrated in Fig. 5. The processed hearing-aid signal $u(n)$ is input to the LP analysis to estimate the all-pole filter $\hat{H}(z)$ and the residual gain $g(n)$ using one of the methods that yield stable models described in Section II-C. The residual gain $g(n)$ approximates the standard deviation of the prediction error/residual signal so that the power of the original signal is maintained. The way of estimating $g(n)$ will be given in Section V. In the LP synthesis stage, a unit-variance white noise excitation $w(n)$ is used to drive the estimated all-pole filter $\hat{H}(z)$ with an amplification of $g(n)$ to produce the synthesize signal $u_{\text {syn }}(n)$, which is high-pass filtered afterwards to obtain the high-frequency component $u_{\text {syn_hp }}(n)$. In the end, $u_{\text {syn_hp }}(n)$ is added to $u_{l p}(n)$, the low-pass filtered $u(n)$, to obtain a new signal $q(n)$ for the receiver to output, i.e.,

$$
q(n)=u_{l p}(n)+u_{\text {syn_hp }}(n) .
$$

By keeping the low-frequency signal intact, the sound quality is improved significantly at least for speech signal as most energy of the speech signal is concentrated at low frequencies. The BLPC vocoder proposed here actually resembles the RELP vocoder, in which the residual signal below $1 \mathrm{kHz}$ is used as the excitation sequence for the LP synthesis. The differences between the RELP and the BLPC vocoder lie in two aspects: first, RELP typically has a cutoff frequency at $1 \mathrm{kHz}$ while BLPC has a cutoff frequency at $2 \mathrm{kHz}$, which means the sound quality of BLPC below $2 \mathrm{kHz}$ is better than that of RELP; Second, in RELP, the high-frequency signal is restored in some nonlinear manner, typically with a rectifier [14], whereas, BLPC restores it with white noise excitation. This implies that RELP may still recover the formants above $1 \mathrm{kHz}$ to some extent while BLPC lmay distort the formants above $2 \mathrm{kHz}$. However, by keeping the original signal intact below $2 \mathrm{kHz}$, BLPC has already maintained the first formants and most of the second formants of vowels. 


\section{Steady-State Analysis of BLPC-AFC}

The bias of the BLPC-AFC can be calculated from a steadystate analysis of the system by assuming that a Least-Square solution is obtained [cf. (11)]

$$
\begin{aligned}
\operatorname{Bias} & =\mathbf{R}_{\mathbf{q q}}^{-1} \mathbf{r}_{\mathbf{q} x} \\
& =\mathbf{R}_{\mathbf{q q}}^{-1} E\left\{\left[\mathbf{u}_{l p}(n)+\mathbf{u}_{\text {syn_hp }}(n)\right] x(n)\right\} \\
& =\mathbf{R}_{\mathbf{q q}}^{-1} E\left\{\mathbf{u}_{l p}(n) x(n)\right\} \\
\mathbf{q}(n) & =\left[q(n), q(n-1), \cdots, q\left(n-L_{\hat{F}}+1\right)\right]^{T}
\end{aligned}
$$

where the vectors $\mathbf{u}_{l p}(n)$ and $\mathbf{u}_{\text {syn_hp }}(n)$ are defined similarly as in (19). From (17) and (18), the assumption is used that the synthesized signal $u_{\text {syn_hp }}(n)$ generated from a white noise sequence is statistically uncorrelated with the desired input signal $x(n)$.

Equation (18) shows that the high-frequency bias is removed. Although the bias remains at low frequencies, it usually does not result in any problem because the feedback cancellation system, in most cases, handles the low-frequency bias very well but fails to prevent whistling at high frequencies as mentioned in Section III-B.

\section{BAND-LIMITED LPC VOCODER FOR AFC WITH FILTERED-X ADAPTATION}

The proposed BLPC-AFC can be further combined with two adaptive decorrelation filters (ADF) in the feedback cancellation path to reduce the short-time correlation in the high-frequency region and yield more accurate estimate of the feedback path. This combination method is called "BLPC-FxAFC" algorithm.

\section{A. Use of the Filtered-X Adaptation in BLPC-AFC}

The BLPC vocoder helps to remove the long-term bias in the high-frequency region as shown in Section III-C. However, short-term correlation still exists especially for tonal signals, which may lead the system adaptation in a wrong direction when the adaptation algorithm, such as normalized-lease-mean-square (NLMS), uses data within a short observation window. To reduce this short-term correlation, two decorrelation filters can be introduced in the feedback cancellation path.

Suppose the estimated all-pole filter $\hat{H}(z)$ is obtained in the LP analysis stage. The inverse of $\hat{H}(z)$ is an FIR filter, which is also referred to as the prediction error filter (PEF) as mentioned in Section II-B. Denote this PEF as $\hat{A}(z)$, then

$$
\hat{A}(z)=1-\sum_{k=1}^{L} \hat{a}_{k} z^{-k} .
$$

The adaptation of the feedback path model is based on the receiver input signal $q(n)$ and the error signal $e(n)$. If both signals are filtered with a decorrelation filter $\hat{A}(z)$ before entering the adaptation, then a structure identical to filtered-X adaptation ${ }^{3}$

\footnotetext{
${ }^{3}$ It should be noted that $\hat{A}(z)$ is dependent on the characteristics of the incoming signal. Therefore, the two decorrelation filters, which use the coefficients of $\hat{A}(z)$, are actually adaptive. Furthermore, in this paper the term "filtered-X" refers to the structure discussed in [20], [22] instead of the structure proposed in [23]. The two filtered-X structures are not equivalent in terms of bias analysis.
}

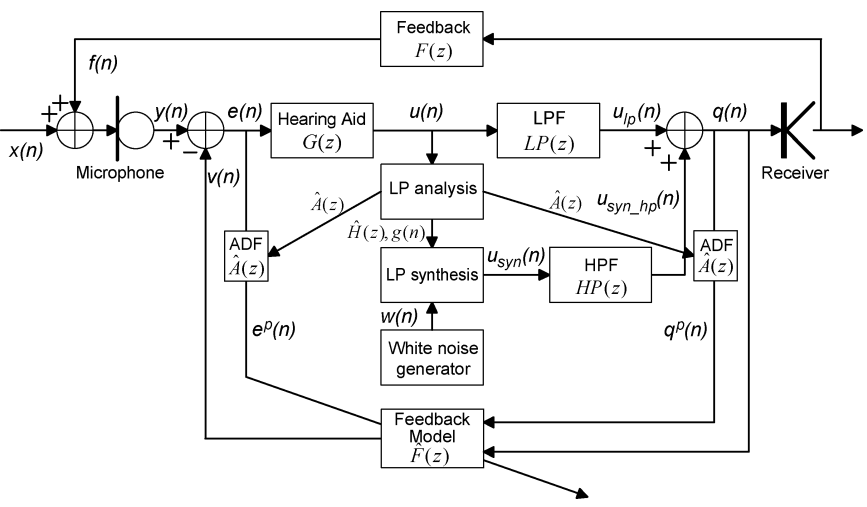

Fig. 6. Diagram of the feedback cancellation system with band-limited LPC vocoder and filtered-X adaptation. The receiver input $q(n)$ and the prediction error signal $q^{\mathrm{p}}(n)$ are both input to the feedback model. The former is used to generate the feedback estimation signal $v(n)$ and the latter is used to update the feedback model together with $e^{\mathrm{p}}(n)$.

is achieved [22]. The advantage of using $\hat{A}(z)$ to filter $q(n)$ and $e(n)$ is that at the receiver end, the high-frequency component of the filtered signal of $q(n)$ will be exactly the high-pass filtered white noise sequence that is used to generate the synthesized signal $u_{\text {syn_hp }}(n)$, i.e., $w(n)$ filtered by $H P(z)$, if the ADFs and the estimated $\hat{A}(z)$ are synchronized perfectly. The temporal correlation between $x(n)$ and $q(n)$ at high frequencies can be decreased significantly in this way, which will be shown by an example in Section V. Since $\hat{H}(z)$ is estimated on the broadband signal $u(n)$, the inverse filter $\hat{A}(z)$ used in the filtered-X will whiten the two signals $x(n)$ and $q(n)$ at low frequencies to some extent ${ }^{4}$ and help to reduce the temporal correlation. The filtered-X adaptation-based BLPC-AFC, BLPC-FxAFC, is illustrated in Fig. 6, where the PEF $\hat{A}(z)$, estimated from LP analysis of the processed hearing-aid signal $u(n)$, is copied to the two ADFs to generate the prediction errors $e^{\mathrm{p}}(n)$ and $q^{\mathrm{p}}(n)$ for adaptation in the feedback model. Since the two ADFs use the same filter $\hat{A}(z)$, the phase misalignment between these two filters is zero and therefore the requirement of phase misalignment for stable adaptation [20] of the filtered-X algorithm is always satisfied. However, due to the group delay associated with the ADFs, the filtered-X algorithm may become unstable if the coefficients of the estimated feedback path $\hat{F}(z)$ change too fast [11].

\section{B. Steady-State Analysis}

In the proposed BLPC-FxAFC, the estimated feedback path in the steady state, assuming that the least-square solution has been obtained, is as follows:

$$
\begin{aligned}
\hat{\mathbf{f}} & =\mathbf{R}_{\mathbf{q}^{\mathrm{p}} \mathbf{q}^{\mathrm{p}}}^{-1} \mathbf{r}_{\mathbf{q}^{\mathrm{p}} y^{\mathrm{p}}} \\
& =\mathbf{R}_{\mathbf{q}^{\mathrm{p}} \mathbf{q}^{\mathrm{p}}}^{-1} \mathbf{r}_{\mathbf{q}^{\mathrm{p}} f^{\mathrm{p}}}+\mathbf{R}_{\mathbf{q}^{\mathrm{p}} \mathbf{q}^{\mathrm{p}}}^{-1} \mathbf{r}_{\mathbf{q}^{\mathrm{p}} x^{\mathrm{p}}} \\
\mathbf{q}^{\mathrm{p}}(n) & =\left[q^{\mathrm{p}}(n), q^{\mathrm{p}}(n-1), \cdots, q^{\mathrm{p}}\left(n-L_{\hat{F}}+1\right)\right]^{T} \\
q^{\mathrm{p}}(n) & =q(n)-\hat{\mathbf{a}}^{T} \mathbf{q}_{L}(n-1) \\
\mathbf{q}_{L}(n) & =[q(n), q(n-1), \cdots, q(n-L+1)]
\end{aligned}
$$

where $\mathbf{R}_{\mathbf{q}^{\mathrm{p}} \mathbf{q}^{\mathrm{p}}}$ is defined similarly as in (12), $\mathbf{r}_{\mathbf{q}^{\mathrm{p}} y^{\mathrm{p}}}, \mathbf{r}_{\mathbf{q}^{\mathrm{p}} f^{\mathrm{p}}}$, and $\mathbf{r}_{\mathrm{q}^{\mathrm{p}} x^{\mathrm{p}}}$ are defined similarly as in (13), the superscript $\mathrm{p}$

\footnotetext{
${ }^{4}$ The low-frequency whitening will not be as effective as that at high frequencies unless the desired input signal $x(n)$ is an AR random process.
} 
denotes the prediction error of the corresponding signal and the prediction errors $y^{\mathrm{p}}(n), x^{\mathrm{P}}(n)$, and $f^{\mathrm{p}}(n)$ are defined similarly as in (24).

In (22), the first term is essentially the steady-state optimal solution to a filtered-X Wiener filtering, which approximates the true feedback path as long as the filter length of $\hat{F}(z)$ is sufficiently large for the feedback path modeling. In the second term, the source of bias $\mathbf{r}_{\mathbf{q}^{\mathrm{p}} x^{\mathrm{p}}}$ and can be further expanded

$$
\begin{aligned}
\mathbf{r}_{\mathbf{q}^{\mathrm{p}} x^{\mathrm{p}}} & =E\left\{\left[\mathbf{u}_{\mathrm{syn} \_\mathrm{hp}}^{\mathrm{p}}(n)+\mathbf{u}_{l p}^{\mathrm{p}}(n)\right] x^{\mathrm{p}}(n)\right\} \\
& =E\left\{\mathbf{u}_{l p}^{\mathrm{p}}(n) x^{\mathrm{p}}(n)\right\}
\end{aligned}
$$

where $\mathbf{u}_{l p}^{\mathrm{p}}(n)$ and $\mathbf{u}_{\mathrm{syn} \_ \text {hp }}^{\mathrm{p}}(n)$ are defined similarly as in (23).

From (26) to (27), the assumption is used that the synthesized signal generated with a white noise sequence is uncorrelated with $x(n)$ and $x^{\mathrm{p}}(n)$.

Equations (26) and (27) show that at high frequencies, the bias can be eliminated as long as the filter length of $\hat{F}(z)$ is sufficient. The feedback estimate at high frequencies is not influenced by the estimation of $\hat{A}(z)$ even when $\hat{A}(z)$ has an insufficient order for modeling $u(n)$ or when it does not model $u(n)$ accurately. 5

It should be noted that the steady-state analysis of the BLPC-AFC in Section III-C has shown similar results, i.e., the elimination of the bias at high frequencies. Therefore, the advantage of the BLPC-FxAFC is not expected in the least-square solution using long-term steady-state data but expected in the practical situation where the adaptation of the feedback model uses data within a short observation window. This will be further explained in Section V-A.

\section{Comparison of BLPC-AFC, BLPC-FxAFC, and PEM-AFC}

Both the BLPC-AFC and the BLPC-FxAFC can eliminate the long-term bias in the high-frequency region as long as the filter $\hat{F}(z)$ is long enough to model the feedback path $F(z)$. The BLPC-FxAFC can further reduce the short-term correlation especially at high frequencies since the prediction error is a high-pass filtered white noise sequence at the receiver end. This can yield a better estimate of the feedback path.

The filtered-X algorithm used in the proposed BLPC-FxAFC is similar to the PEM-AFC proposed in [11] to some extent because both use linear prediction coefficients to decorrelate the error signal and the receiver input signal. The difference lies in the fact that the PEM-AFC uses the linear prediction at the microphone end, whereas the BLPC-FxAFC uses the linear prediction at the receiver end. If it is assumed that the forward-path hearing aid process $G(z)$ contains only a delay $d_{G}$ and a constant linear amplification $g_{G}$, the position of linear prediction after the hearing-aid process does not result in any difference in the steady-state performance [24]. In this sense, the proposed BLPC-FxAFC can also be roughly interpreted as the combination of a BLPC-AFC with reduced short-term correlation in the high-frequency region and a modified PEM-AFC in the low-frequency region.

\footnotetext{
${ }^{5}$ The under-modeling or wrong modeling does not introduce any bias but will degrade sound quality of the synthesized signal.
}

The PEM-AFC removes the bias only when the desired input signal $x(n)$ is an AR random process and when certain conditions are met [11]. For a large set of real-life signals, such as voiced speech and tonal music, which can hardly be modeled by an all-pole filter, the PEM-AFC still suffers from a biased solution because the prediction error signals $u^{\mathrm{p}}(n)$ and $e^{\mathrm{p}}(n)$ are not white [11]. Moreover, under-modeling of the desired input signal $x(n)$ may also introduce bias into the estimation. For these two cases, the BLPC-FxAFC can still be useful in removing the bias in the high-frequency region where feedback usually occurs.

\section{Simulations AND DiscUSSION}

To evaluate and compare the performance of the algorithms, simulations are carried out for AFC, BLPC-AFC, PEM-AFC, Filtered-X AFC (FxAFC), and BLPC-FxAFC. The FxAFC uses the same filtered-X approach as used in the BLPC-FxAFC but does not involve the synthesis stage. It can also be regarded as a modified PEM-AFC with linear prediction placed at the receiver end.

The five methods are simulated with a sampling frequency of $16 \mathrm{kHz}$. The processing is carried out on a block by block basis with a block size of 24 samples, corresponding to $1.5 \mathrm{~ms}$. The forward path $G(z)$ consists of a delay $d_{G}$ of 24 samples and an adjustable linear gain $g_{G}$. Most hearing impaired people have greater hearing loss at high frequencies. Therefore, the prescribed gain in the forward path will also be higher at high frequencies. This gain setting has become one of the biggest challenges for feedback cancellation in practice. To simulate a realistic gain setting in the hearing aids and also to test the performance of the algorithms with high gains at frequencies where feedback oscillation usually occurs, the forward-path gain $g_{G}$ is set to $15 \mathrm{~dB}$ at frequencies below $2 \mathrm{kHz}$ and $35 \mathrm{~dB}$ above $2 \mathrm{kHz}$ in all the simulations.

In the simulations, the feedback path is an FIR filter of order 50 obtained from the measurement of a commercial behindthe-ear (BTE) hearing aid, ReSound Metrix MX70-DVI. The frequency response of the feedback path is illustrated in Fig. 7, which has large magnitude responses from 2 to $7 \mathrm{kHz}$. The maximum stable gain without feedback canceller is around $15 \mathrm{~dB}$ at $3.3 \mathrm{kHz}$. The feedback model $\hat{F}(z)$ consists of an adaptive FIR filter of 50 orders, which is initialized as the true feedback path to show how the estimate of the feedback path drifts away from the true feedback path due to the bias problem. This initialization of the filter is also considered as a result of a common fitting procedure for the feedback cancellation in the industry [3], in which the true feedback path is measured and used as the starting point and/or constraint of the adaptation.

The adaptive filter is updated by a block-based NLMS algorithm, which is a modified block LMS algorithm [15]. In the $\mathrm{AFC}$ and BLPC-AFC, the update is performed as follows:

$$
\begin{aligned}
\hat{\mathbf{f}}(m+1) & =\hat{\mathbf{f}}(m)+\alpha(m) \sum_{i=0}^{B-1} \mathbf{q}(m B+i) e(m B+i) \\
\alpha(m) & =\frac{\mu}{\delta+\sum_{i=0}^{i=B-1}|q(m B+i)|^{2}}
\end{aligned}
$$



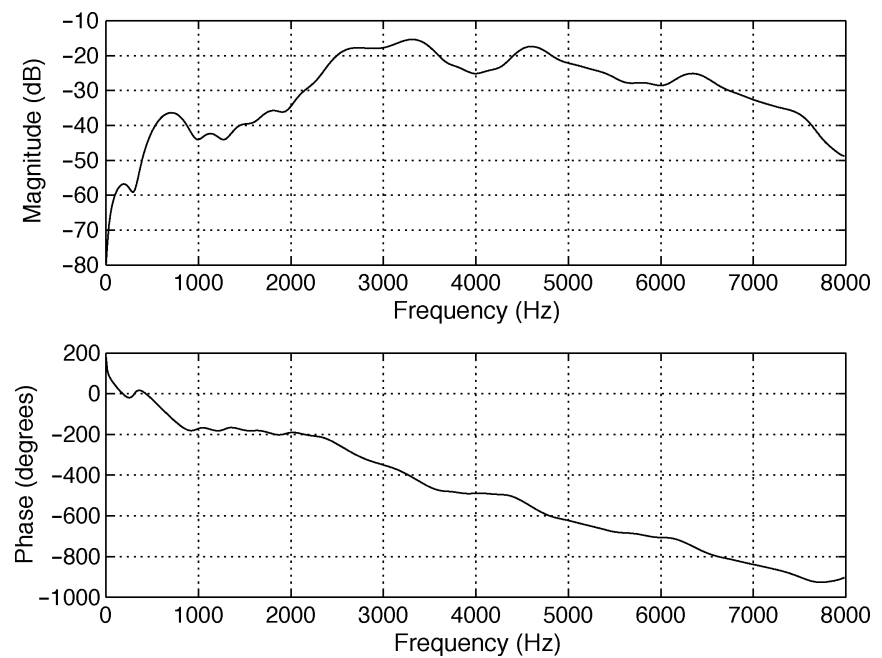

Fig. 7. Frequency response of the feedback path of 50 orders based on the measurement of a commercial BTE hearing aid: ReSound Metrix.

where $\mathbf{q}(m B+i)$ is defined in (19), $m$ is the block index, $B$ is the block size and equals $24, \mu$ is the step-size parameter and set to 0.002 in the simulations, and $\delta$ is set to a small positive constant to overcome numerical difficulties. For PEM-AFC, FxAFC, and BLPC-FxAFC, the update is similar

$$
\begin{aligned}
\hat{\mathbf{f}}(m+1)= & \hat{\mathbf{f}}(m) \\
& +\alpha(m) \sum_{i=0}^{B-1} \mathbf{q}^{\mathrm{p}}(m B+i) e^{\mathrm{p}}(m B+i) \\
\alpha(m)= & \frac{\mu}{\delta+\sum_{i=0}^{i=B-1}\left|q^{\mathrm{p}}(m B+i)\right|^{2}}
\end{aligned}
$$

where $\mathbf{q}^{\mathrm{p}}(m B+i)$ is defined in (23), AND $q^{\mathrm{p}}(m B+i)$ is defined in (24).

In PEM-AFC, FxAFC, and BLPC-FxAFC, the PEF $\hat{A}(z)$ are of the length 21, which is the same as used in [11]. The autocorrelation method Levinson-Durbin algorithm, which yields stable models, is used with an analysis window length of 10.5 ms, corresponding to 168 samples or 7 blocks. The $\hat{H}(z)$ is updated for every new block. Therefore, the linear prediction for the current block is based on the data in the current block and in the six previous blocks. The residual gain for block $m$ is estimated in the following way:

$$
g(m)=\sqrt{\frac{1}{B} \sum_{i=0}^{B-1}\left|u^{\mathrm{p}}(m B+i)\right|^{2}}
$$

where $u^{\mathrm{p}}(m B+i)$ is defined similarly as in (24), and $\hat{\mathbf{a}}(m)$ is the estimated coefficients of the all-pole model at block $m$. The residual gain $g(m)$ makes sure that the power of the residual signal $u^{\mathrm{p}}(n)$ in each block is the same as the variance of the noise sequence used for synthesis, which is done in the way as illustrated in Fig. 2:

$u_{\text {syn }}(m B+i)=\hat{\mathbf{a}}(m)^{T} \mathbf{u}_{s y n, L}(m B+i-1)+g(m) w(m B+i)$

where $i=0,2, \cdots, B-1$ and $\mathbf{u}_{s y n, L}(m B+i-1)$ is defined similarly as in (25).

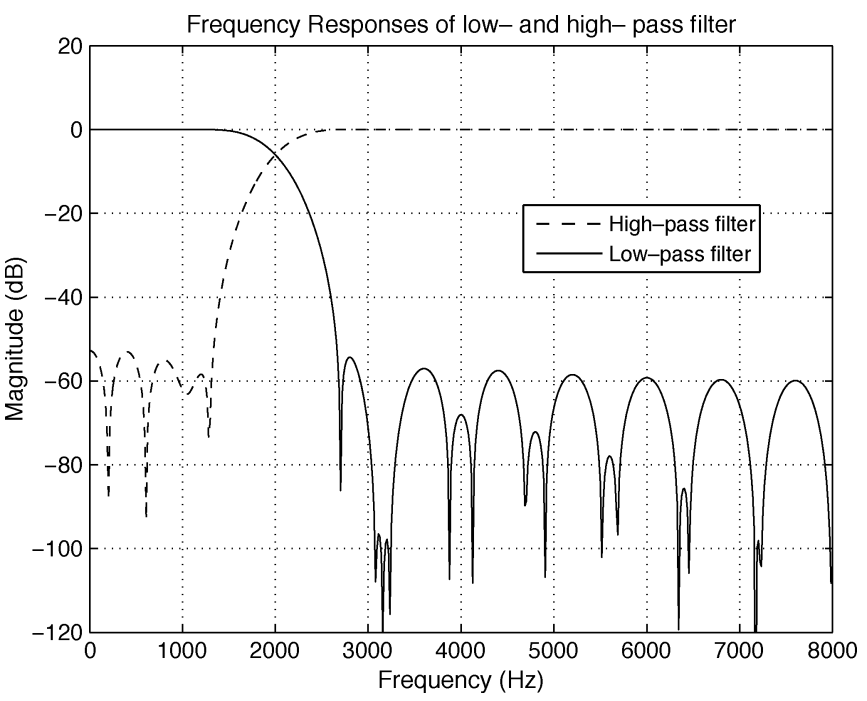

Fig. 8. Frequency responses of the complimentary low- and high-pass filters.

The high-pass filter $H P(z)$ is a 40-order FIR filter and has a cutoff frequency of $2 \mathrm{kHz}$. It is designed with the classical windowed linear-phase FIR digital filter design method [25] using a hamming window. The low-pass filter $L P(z)$ is also 40-order and is the strict complementary filter of $\operatorname{HP}(z)$, i.e.,

$$
L P(z)=z^{-n_{0}}-H P(z)
$$

where $n_{0}$ is the group delay of the designed $H P(z)$ and equals 20 samples. The additional delay introduced by $n_{0}$ in the forward path of BLPC-AFC and BLPC-FxAFC is accordingly added in the forward path of AFC, PEM-AFC, and FxAFC so that the performance comparison between these algorithms is not influenced by the overall forward-path delay. The overall forward-path delay therefore is the sum of $d_{G}$ and $n_{0}$ for all the algorithms. The frequency responses of the low- and high-pass filters are shown in Fig. 8.

The performance of the algorithms is evaluated by the misalignment between the true feedback path $F(z)$ and the modeled feedback path $\hat{F}(z)$. The misalignment is calculated at frequencies above $2 \mathrm{kHz}$ to quantify the modeling error in the critical frequency region where feedback oscillation usually occurs and to show the effects of the BLPC vocoder. The misalignment above $2 \mathrm{kHz}$ is denoted as $\varsigma$, which is computed in the frequency domain as

$$
\begin{aligned}
\varsigma & =\frac{\sum_{k=N_{0}}^{N_{f}-1}\left|F\left(e^{j 2 \pi\left(k / N_{f}\right)}\right)-\hat{F}\left(e^{j 2 \pi\left(k / N_{f}\right)}\right)\right|^{2}}{\sum_{k=N_{0}}^{N_{f}-1}\left|F\left(e^{j 2 \pi\left(k / N_{f}\right)}\right)\right|^{2}} \\
N_{0} & =\left\lceil 2000 /\left(\mathrm{f}_{\mathrm{s}} / N_{f}\right)\right\rceil
\end{aligned}
$$

where $\lceil\cdot\rceil$ is the ceiling function to get the smallest integer not less than the value in the brackets, $N_{f}$ equals the number of frequency points, which is 1024 in this paper, and $\mathrm{f}_{\mathrm{s}}$ is the sampling frequency, which is equal to $16000 \mathrm{~Hz}$ in our simulation. Therefore, $N_{0}$ is calculated as 128 . 


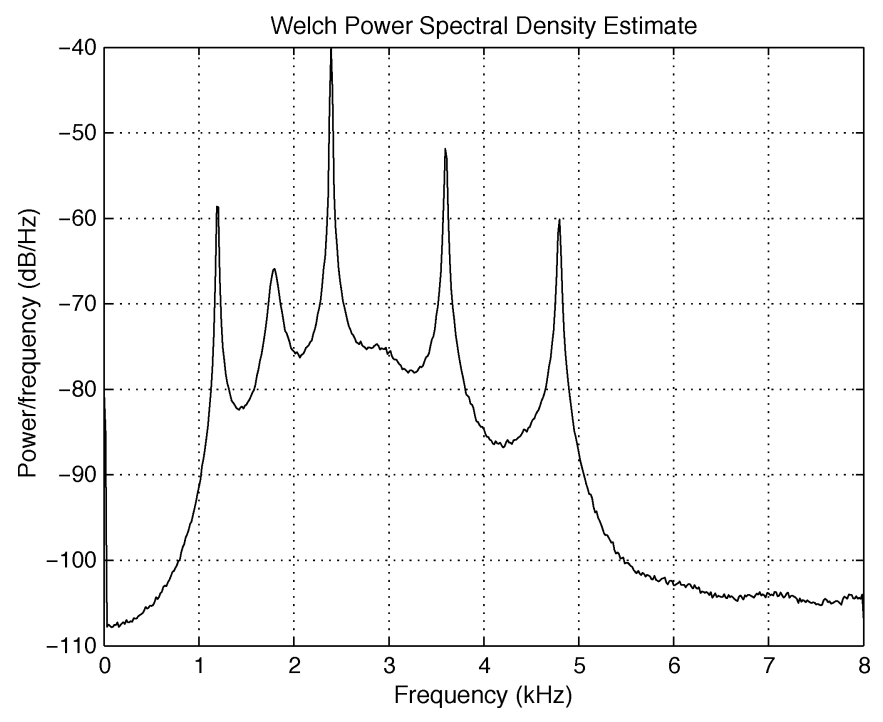

Fig. 9. Power spectral density of the 20-order AR random process.

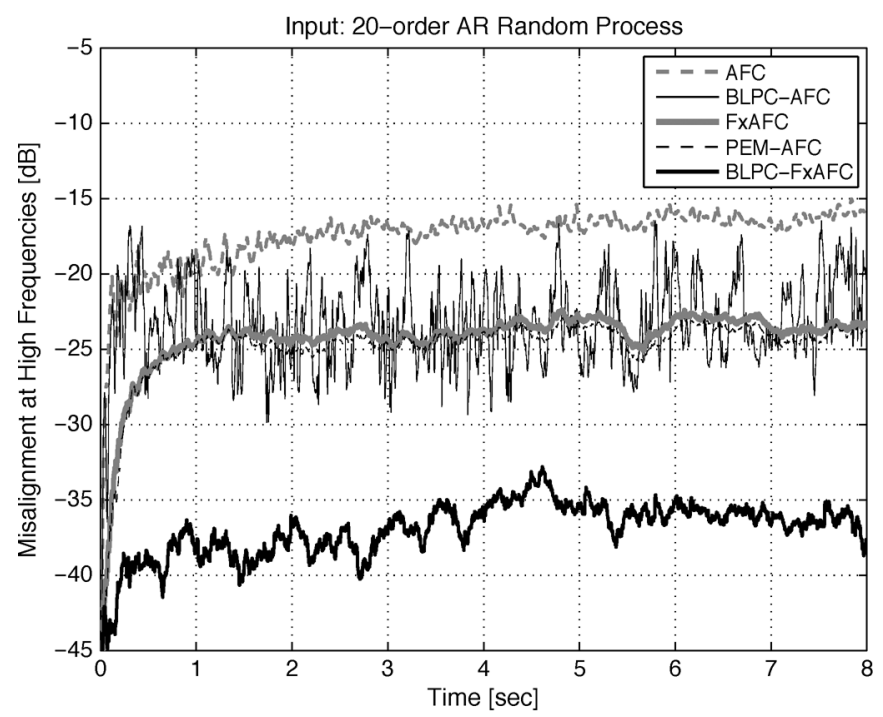

Fig. 10. Misalignment at high frequencies when a 20-order AR random process is used as the desired input signal.

\section{A. Simulation Results With a Stationary AR Signal Input}

To examine the performance of the algorithms, a stationary $\mathrm{AR}$ random process of $8 \mathrm{~s}$, which is generated by a 20 -order allpole filter $H_{1}(z)$, also called "signal model," is used as the input signal in the first test case. The power spectrum density (PSD) of the AR signal, which is shown in Fig. 9, exhibits sharp peaks. Therefore, the bias problem is expected to be serious for the conventional AFC. The misalignment above $2 \mathrm{kHz}$ is depicted in Fig. 10.

As can be seen from Fig. 10, due to the bias problem, the AFC exhibits the largest misalignment. The BLPC-AFC lowers the misalignment by around $7 \mathrm{~dB}$ on average. However, its misalignment has the largest fluctuations because the short-term correlation between the synthesized AR signal and the original AR signal has a large variance.

To illustrate this short-term correlation, suppose there are two AR signals $r_{1}(n)$ and $r_{2}(n)$ generated from the same signal
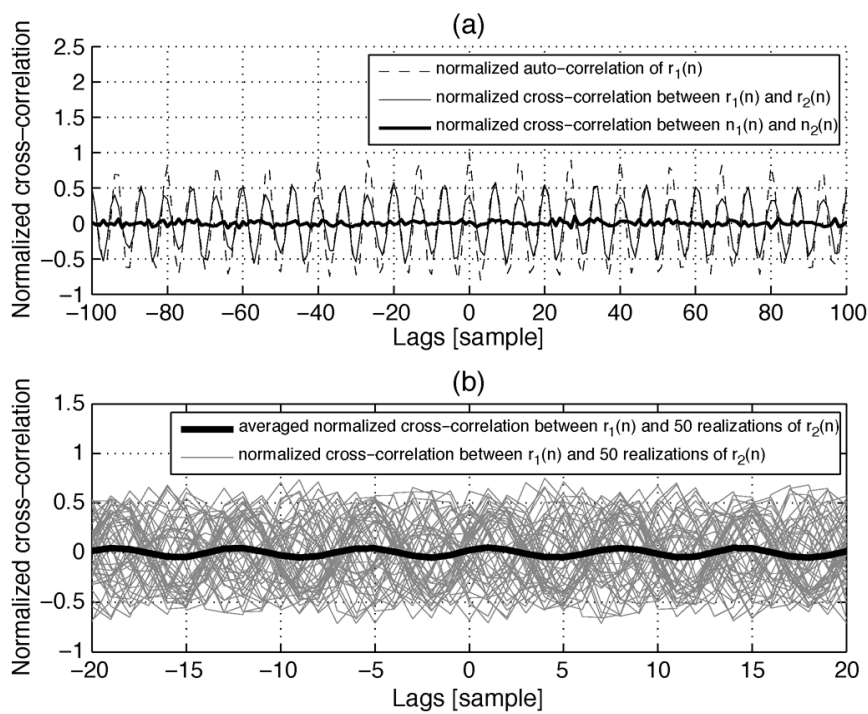

Fig. 11. (a) Normalized cross-correlation between two 20-order AR random processes $r_{1}(n)$ and $r_{2}(n)$, between their corresponding white noise sequences $n_{1}(n)$ and $n_{2}(n)$, and the auto-correlation of $r_{1}(n)$. (b) The normalized crosscorrelation between $r_{1}(n)$ and 50 realizations of $r_{2}(n)$ and the averaged normalized cross-correlation.

model as that of the test signal, i.e., $H_{1}(z)$, but with two different white noise sequences $n_{1}(n)$ and $n_{2}(n)$, respectively. The two signals are both of the length 1000 samples. The normalized cross-correlation ${ }^{6}$ of $r_{1}(n)$ and $r_{2}(n)$, auto-correlation of $r_{1}(n)$ and cross-correlation of $n_{1}(n)$ and $n_{2}(n)$ are illustrated in Fig. 11(a). As shown in the figure, the decaying speed of the auto-correlation of $r_{1}(n)$ is very slow and therefore the short delay in the hearing-aid forward path is not sufficient to reduce the correlation between $r_{1}(n)$ and its delayed replica. When the delayed replica of $r_{1}(n)$ is replaced by another uncorrelated AR process $r_{2}(n)$, the short-term correlation gets smaller but is still high. However, the cross-correlation between $n_{1}(n)$ and $n_{2}(n)$ is much smaller. This explains why the BLPC-FxAFC yields much better performance than the BLPC-AFC in Fig. 10. The temporal correlation between $r_{1}(n)$ and $r_{2}(n)$ exhibits a very large variance as shown in Fig. 11(b), where the cross-correlation between $r_{1}(n)$ and 50 realizations of $r_{2}(n)$ and the averaged cross-correlation are illustrated. The 50 realizations of $r_{2}(n)$ are obtained by using 50 different white noise sequences. The large variance of the temporal correlation between $r_{1}(n)$ and $r_{2}(n)$ results in large fluctuations in the misalignment curve of the BLPC-AFC. It can also be seen that the average cross-correlation is much smaller, which implies that the long-term bias can be removed by the BLPC-AFC.

Fig. 10 also shows that the performance of the FxAFC is very close to that of the PEM-AFC, and the performance of the BLPC-FxAFC is much better than that of the FxAFC and PEM-AFC. This is because the online estimation of the signal model from a short observation window exhibits variation, which will result in nonwhite prediction error and short-term bias, and therefore limits the performance of the FxAFC and

${ }^{6}$ The normalized cross-correlation refers to the cross-correlation between two normalized sequences. Each sequence is normalized so its autocorrelation at zero lag is unity. 


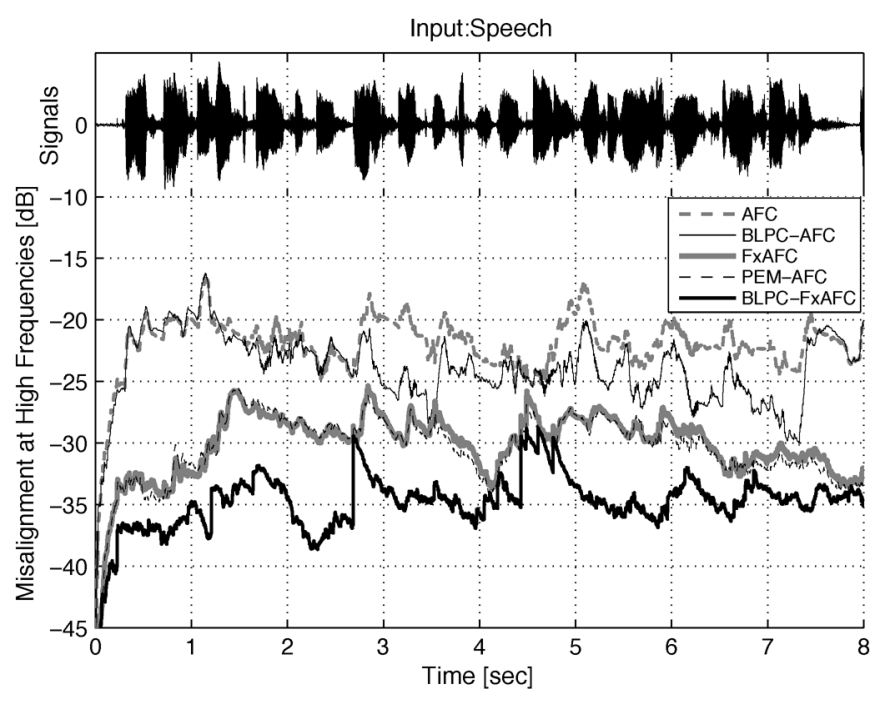

Fig. 12. Speech signal and the misalignments at high frequencies.

PEM-AFC. For the BLPC-FxAFC, although this problem also exists, the performance is not influenced too much because the prediction error at the receiver end is always a high-pass filtered white noise sequence as pointed out in Section IV-A.

\section{B. Simulation Results With a Speech Signal Input}

In the second test case, an 8-s sample of female speech is used as the input signal. The speech signal and the misalignment above $2 \mathrm{kHz}$ is illustrated in Fig. 12.

Fig. 12 shows that the misalignment of the BLPC-AFC is around $2-3 \mathrm{~dB}$ lower than that of the AFC. The performance of the FxAFC is again very close to that of the PEM-AFC. Compared with the FxAFC and PEM-AFC, the BLPC-FxAFC reduces the misalignment by around $5 \mathrm{~dB}$ on average. This shows that the BLPC vocoder helps to improve the estimation accuracy of the feedback path. The filtered-X based algorithms yield better performance as expected.

The difference in the performance between the best and the worst algorithms is smaller than that in the previous test case. This is because the speech signal is generally not very correlated with itself. Although during the periods of voiced speech the autocorrelation is significant, the voiced state usually does not last very long, and thus the buildup of bias is not large if the stepsize parameter of the adaptation in the feedback cancellation is small enough and/or when a sufficient delay is introduced in the hearing-aid process. Therefore, the bias problem tends to be smaller with speech input signal.

It can also be noticed that all the curves exhibit significant fluctuations. This is due to the dynamic nature of speech. The speech signal is only stationary for $10-20 \mathrm{~ms}$ and switches frequently between voiced state, unvoiced state and pauses.

The misalignment of the FxAFC, PEM-AFC, and BLPC-FxAFC fluctuates more than that of the AFC and BLPC-AFC. This actually happens in the transient part of speech, during which the analysis frame of linear prediction contains a segment of nonstationary signal. Linear prediction with nonstationary data will result in an inaccurate model. For

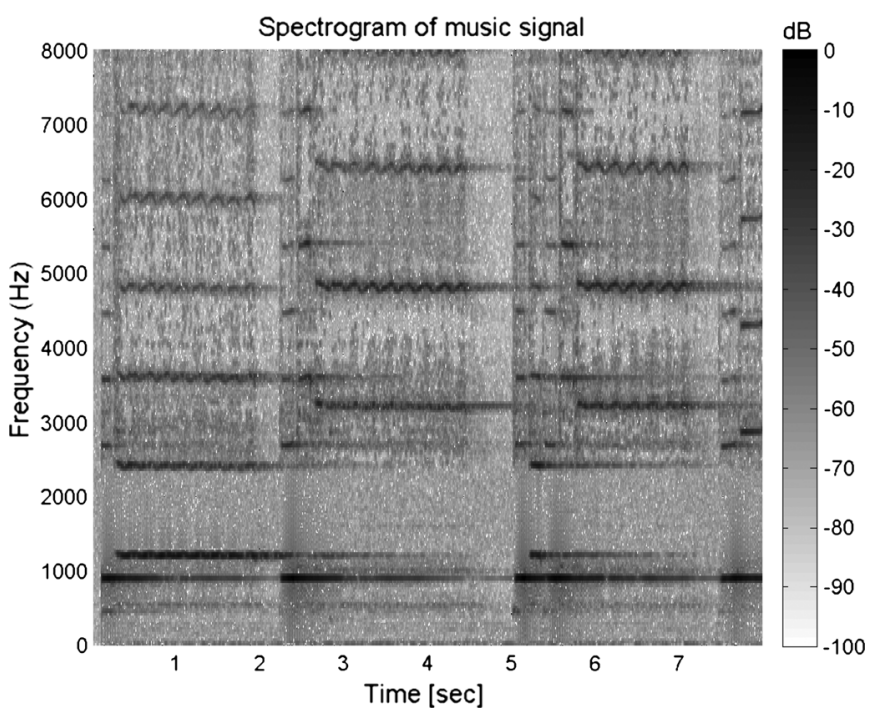

Fig. 13. Spectrogram of the 8-s flute music signal which is normalized so that the maximum peak is $0 \mathrm{~dB}$.

the FxAFC and PEM-AFC, using the inverse of this inaccurate model as the ADFs does not whiten $e(n)$ at the microphone side and $q(n)$ at the receiver input, and may even color the signal and introduce short-term bias in the adaptation. For the BLPC-FxAFC, this inaccurate modeling also occurs, but the misalignment is smaller than the FxAFC and PEM-AFC because at the receiver end the signal after the decorrelation filter $q^{\mathrm{P}}(n)$ is white at high frequencies.

\section{Simulation Results With a Music Signal Input}

In the third case, an 8-s sample of flute music is used as the input signal. The spectrogram of the music signal is illustrated in Fig. 13, which shows that the music signal is very tonal and therefore very challenging for feedback cancellation systems. The spectrogram is normalized so that the maximum magnitude is $0 \mathrm{~dB}$.

The misalignment above $2 \mathrm{kHz}$ is shown in Fig. 14. The BLPC-AFC and AFC both yield large misalignment although the BLPC-AFC is slightly better. This is because the short-time correlation for the tonal flute music input is very high even when the original signal is replaced by the synthesized signal generated with a white noise sequence (cf. Fig. 11). It takes a long time to average out this high temporal correlation with an NLMS adaptation algorithm. In fact, feedback whistling happens for the AFC and the BLPC-AFC at some places of the output signal.

The performance of the FxAFC and PEM-AFC is very similar. Thanks to the two ADFs, they both give a better performance than the AFC and the BLPC-AFC. But the remaining bias still exists because the flute signal is not a perfect AR process. The BLPC-FxAFC shows a significant improvement in the performance over the other three methods because of both the replacement with an uncorrelated signal and the filtered- $X$ adaptation.

\section{Remarks on Sound Quality}

The sound quality of the synthetic signals using the BLPC with the same parameters and linear prediction algorithm 


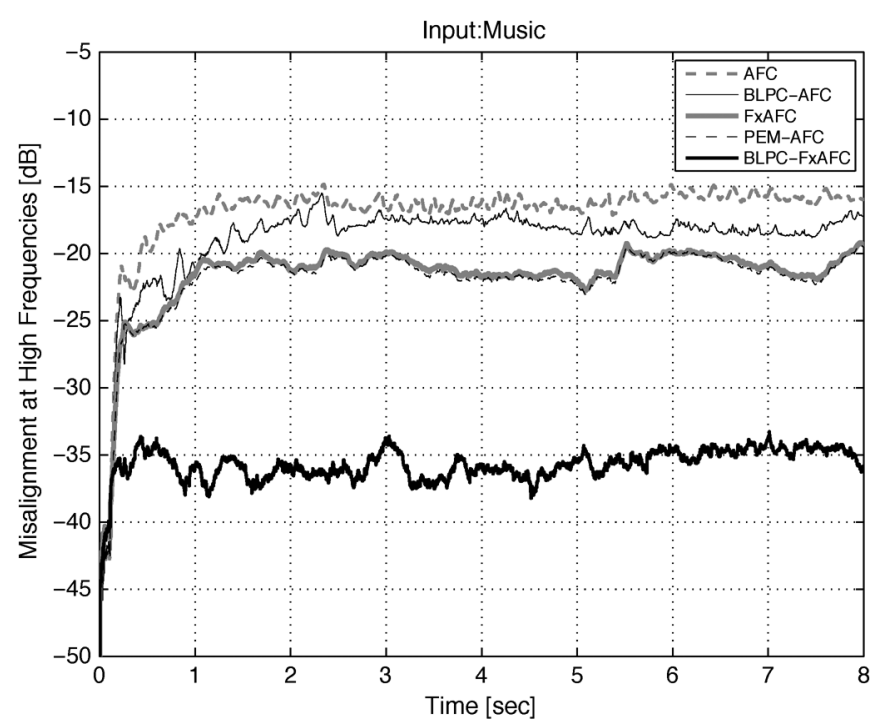

Fig. 14. Misalignment at high frequencies when the flute music is used as the desired input signal.

(Levinson-Durbin) in the simulation has been evaluated subjectively by the authors.

For speech samples, the overall sound quality is degraded very little although the difference between the original speech and the synthesized speech can still be perceived. For hearing impaired listeners, it is very likely that even this difference can hardly be detected. During the transient part of speech, noticeable effects due to the inaccurate modeling as mentioned in Section V-B are very rare. This is mainly because of the characteristics and the parameters of the BLPC. First, a relatively short analysis window ( $\sim 10 \mathrm{~ms}$ ) with heavy overlapping $(85.7 \%)$ is used in the BLPC to get a good time resolution, which is one of the easiest ways to reduce transient effect [26]. Second, the BLPC synthesis only takes effect at high frequencies and therefore the dominant energy of speech, which is usually located at low frequencies, may partially mask the error signal resulted from the inaccurate modeling in the high-frequency region. Last, since the synthesis is driven by a white noise sequence instead of an impulse train, only noise could be heard when inaccurate modeling happens instead of other unpleasant artifacts. When the microphone noise and ambient noise are present, this noise due to inaccurate modeling sounds even weaker or inaudible.

For tonal music samples, the degradation of sound quality depends on the characteristics of the signals. For signals with a few sharp peaks spaced sparsely in the high-frequency spectrogram, such as the flute music sample, although the sound quality is not preserved as well as for speech, it is not degraded very much due to a high-order all-pole filter used to model the spectrogram. For signals with a lot of peaks at high frequencies of the spectrogram or very complicated high-frequency spectrogram, the sound quality is degraded to some extent because the modeling fails to capture the spectrogram.

The thorough evaluation of the sound quality is not the scope of this paper, which aims at technical description of the algorithms and performance evaluation. The perceptual validity of these preliminary findings is best addressed using a clinical trial or/and an objective measure which will be the subject of future studies.

\section{CONClusion AND Future DiRECTIONS}

In this paper, a new approach to the bias problem encountered in adaptive feedback cancellation in hearing aids is presented. The main idea of the method is to replace the receiver input signal with a synthesized signal, which sounds perceptually similar to or even identical to the original signal but is statistically uncorrelated with the desired input signal.

To achieve this, a BLPC vocoder is proposed, which is based on band-limited linear predictive coding of the processed hearing-aid signal. To obtain effective decorrelation, impulse trains are not used for excitation as in conventional LPC-based vocoders during voiced speech. Instead, a white noise sequence is always used to drive the estimated signal model to generate the synthesized signal. Based on the facts that the magnitude of the frequency response of the feedback path is usually much higher in the high-frequency region and that the AFC usually breaks down at high frequencies, the signal replacement is performed at high frequencies to focus on the critical frequency region to improve the performance of the AFC and also to reduce the degradation in sound quality.

The BLPC vocoder can be used on top of a conventional AFC to yield the BLPC-AFC, which reduces the long-term bias. Moreover, the BLPC-AFC method can be further combined with filtered-X adaptation to get the BLPC-FxAFC, which can effectively reduce the short-term bias. The proposed BLPC-FxAFC can also be regarded as a modified version of the previously proposed PEM-AFC approach combined with the BLPC vocoder.

The simulation results show that the BLPC is effective in reducing the bias and the misalignment between the estimated and the real feedback paths. The BLPC-FxAFC method has the best performance for all the test signals.

The BLPC vocoder has a cutoff frequency at $2 \mathrm{kHz}$, which avoids severe degradation of sound quality. According to the subjective evaluation of the authors, the sound quality is very well preserved for speech. For many music signals with only a few peaks sparsely spaced at high-frequency spectrogram, the sound quality is not degraded very much either. A clinical trial and/or objective measure is still needed in the future to verify these findings, which will be the subject of future research. In addition, it is found that the dynamic nature of speech makes it hard for the prediction error filter to keep up with and to effectively decorrelate the signals. Two possible approaches could be investigated to improve the dynamic AR modeling in the future: the first approach is to use other time-varying LPC techniques, such as the methods proposed in [27]; the second approach is to use a detector of speech transition to adjust the position and length of the analysis window of linear prediction.

\section{ACKNOWLEDGMENT}

The authors would like to thank the reviewers for their valuable suggestions and comments. 


\section{REFERENCES}

[1] B. Rafaely, M. Roccasalva-Firenze, and E. Payne, "Feedback path variability modeling for robust hearing aids," J. Acoust. Soc. Amer., vol. 107 , no. 5, pp. 2665-2673, 2000.

[2] S. F. Lybarger, "Acoustic feedback control," in The Vanderbilt Hearing-Aid Report, Studebaker and Bess, Eds. Upper Darby, PA: Monographs in Contemporary Audiology, 1982, pp. 87-90.

[3] J. M. Kates, "Constrained adaptation for feedback cancellation in hearing aids," J. Acoust. Soc. Amer., vol. 106, no. 2, pp. 1010-1019, 1999.

[4] M. G. Siqueira and A. Alwan, "Steady-state analysis of continuous adaptation in acoustic feedback reduction systems for hearing-aids," IEEE Trans. Speech Audio Process., vol. 8, no. 4, pp. 443-453, Jul. 2000.

[5] M. A. Stone and B. C. J. Moore, "Tolerable hearing aid delays. i. estimation of limits imposed by the auditory path alone using simulated hearing losses," Ear Hear., vol. 20, no. 3, pp. 182-191, 1999.

[6] H. A. L. Joson, F. Asano, Y. Suzuki, and S. Toshio, "Adaptive feedback cancellation with frequency compression for hearing aids," J. Acoust. Soc. Amer., vol. 94, no. 6, p. 3248C3254, 1993.

[7] C. Boukis, D. P. Mandic, and A. G. Constantinides, "Toward bias minimization in acoustic feedback cancellation systems," J. Acoust. Soc. Amer., vol. 121, no. 3, pp. 1529-1237, 2007.

[8] H. R. Skovgaard, "Hearing Aid Compensating for Acoustic Feedback," U.S. patent 5,680,467, 1997.

[9] J. Hellgren and U. Forssell, "Bias of feedback cancellation algorithms in hearing aids based on direct closed loop identification," IEEE Trans. Acoust., Speech, Signal Process., vol. 9, no. 8, pp. 906-913, 2001.

[10] N. A. Shusina and B. Rafaely, "Feedback cancellation in hearing aids based on indirect close-loop identification," in Proc. IEEE Benelux Signal Process. Symp, 2002, pp. 177-180.

[11] A. Spriet, I. Proudler, M. Moonen, and J. Wouters, "Adaptive feedback cancellation in hearing aids with linear prediction of the desired signal," IEEE Trans. Signal Process., vol. 53, no. 10, pp. 3749-3763, Oct. 2005.

[12] B. S. Atal, "The history of linear prediction," IEEE Signal Process. Mag., vol. 23, no. 2, pp. 154-161, Apr. 2006.

[13] T. E. Tremain, "The government standard linear predictive coding algorithm: Lpc10," Speech Technol., vol. 1, pp. 40-49, 1982.

[14] J. R. Deller, J. G. Proakis, and J. H. Hansen, Discrete-Time Processing of Speech Signals. New York: Macmillan, 1993.

[15] S. Haykin, Adaptive Filter Theory, 4th ed. Upper Saddle River, NJ: Prentice-Hall, 2002.

[16] L. R. Rabiner and R. W. Schafer, Digital Processing of Speech Signals. Englewood Cliffs, NJ: Prentice-Hall, 1978.

[17] N. Levinson, "The wiener rms (root-mean-square) error criterion in filter design and prediction," J. Math. Phys., vol. 25, pp. 261-278, 1947.

[18] J. Durbin, "The fitting of time series models," Rev. Inst. Int. Statist., vol. 28, pp. 233-243, 1960 .

[19] J. P. Burg, "Maximum entropy spectral analysis," in Proc. 37th Meeting Soc. Explor. Geophys., 1967.

[20] H. F. Chi, S. X. Gao, S. D. Soli, and A. Alwan, "Band-limited feedback cancellation with a modified filtered-x LMS algorithm for hearing aids," Speech Commun., vol. 39, no. 1, pp. 147-161, 2003.

[21] J. M. Kates, "Feedback Cancellation in a Hearing Aid With Reduced Sensitivity to Low-Frequency Tonal Inputs," U.S. patent, US 6,831,986, 2004

[22] E. Bjarnason, "Analysis of the filtered-x LMS algorithm," IEEE Trans. Speech Audio Process., vol. 3, no. 6, pp. 504-514, Nov. 1995.

[23] B. Widrow and S. Stearns, Adaptive Signal Processing. Englewood Cliffs, NJ: Prentice-Hall, 1985.

[24] M. M. Yasmin and M. B. J. Carlos, "Mean weight behavior of coupled LMS adaptive systems applied to acoustic feedback cancellation in hearing aids," in Proc. ICISP, 2008, pp. 527-535.

[25] Programs for Digital Signal Processing. New York: IEEE Press, 1979, Digital Signal Processing Committee.
[26] L. H. Zetterberg and Q. Zhang, "Elimination of transients in lpc based vocoders," in Proc. IEEE Int. Symp. Circuits Syst., 1988, pp. 1079-1082.

[27] M. G. Hall, A. V. Oppenheim, and A. S. Willsky, "Time-varying parametric modeling of speech," Signal Process., vol. 5, no. 3, pp. 267-285, 1983.

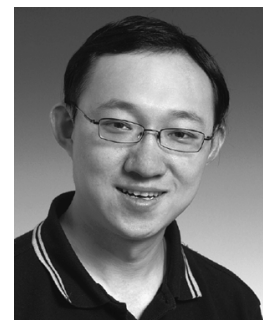

Guilin Ma (M'10) received the B.Sc. degree in electrical engineering from Southeast University, Nanjing, China, in 2002 and the M.Sc. and Ph.D. degrees in acoustic technology, electrical engineering from the Technical University of Denmark, Lyngby, in 2006 and 2010, respectively.

He joined GN ReSound A/S, Ballerup, Denmark, in 2004 and currently works as a Research Scientist. His research interests include adaptive signal processing and acoustical modeling.

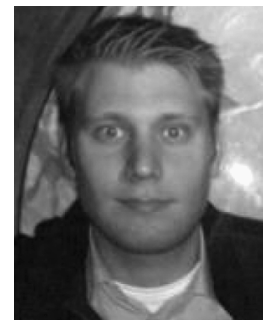

Fredrik Gran received the M.Sc. degree in engineering physics from Lund University, Lunf, Sweden, in 2002 and the Ph.D. degree from the Technical University of Denmark, Lyngby, for work on ultrasound signal processing.

From 2005 to 2008, he was an Assistant Professor at the Technical University of Denmark. In January 2008, he joined GN ReSound A/S, Ballerup, Denmark, as a Research Scientist in hearing aid signal processing. His research interests include adaptive signal processing, adaptive beamforming,

and acoustics.

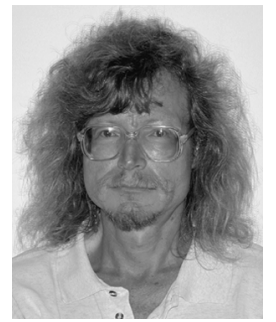

Finn Jacobsen received the M.Sc. degree in electronic engineering and the Ph.D. degree in acoustics from the Technical University of Denmark, in 1974 and 1981, respectively.

His research interests include general linear acoustics, acoustic measurement techniques and signal processing, transducer technology, and statistical methods in acoustics. He has published more than 70 papers in refereed journals and more than 80 conference papers.

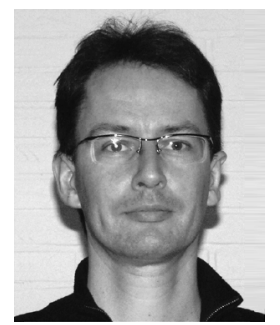

Finn Thomas Agerkvist received the M.Sc. degree in electrical engineering and the Ph.D. degree from the Technical University of Denmark, Lyngby, in 1991 and 1994, respectively.

From 1994 to 1997, he was an Assistant Professor in the Department of Acoustic Technology, Technical University of Denmark. From 1997 to 2001, he was a Senior Scientist at the Danish Defence Research Establishment. Since 2002, he has been an Associate Professor in the Department of Acoustic Technology, DTU-Elektro, Technical University of Denmark. His research interests include signal processing, electro-acoustics, and nonlinear systems. 